\title{
Lung re-inflation after one-lung ventilation for thoracic surgery: an alternative technique
}

\author{
Jean S. Bussières, MD • Paula A. Ugalde, MD
}

Received: 18 November 2014/ Accepted: 8 December 2014/Published online: 1 January 2015

(C) Canadian Anesthesiologists' Society 2014

\section{To the Editor,}

We read with great interest the correspondence from Dr. Bruin $^{1}$ concerning the importance of clamping the dependent lung lumen of the double-lumen endobronchial tube and suspending ventilation of the non-operative lung when the non-dependent lung, having just undergone surgery, is to be re-inflated.

As mentioned by Dr. Bruin, clamping the dependent lung during re-expansion of the non-dependent lung protects it against volutrauma and avoids possible pulmonary tamponade and secondary hypotension. ${ }^{2} \mathrm{We}$ prefer, however, to use a secondary auxiliary breathing circuit to ventilate the non-dependent lung manually. This method allows us to take the time needed to overcome atelectatic areas slowly and progressively ${ }^{3,4}$ and to check for air leaks with minimal risk of staple line disruption. Most importantly, the dependent lung remains ventilated with the same one-lung ventilation parameters.

J. S. Bussières, MD ( $\square)$

Department of Anesthesiology and Critical Care, Université

Laval, Quebec City, QC, Canada

e-mail: jbuss@criucpq.ulaval.ca

\section{J. S. Bussières, MD}

Department of Anesthesiology, Institut universitaire de cardiologie et de pneumologie de Québec, Quebec City, QC, Canada

\section{P. A. Ugalde, MD}

Department of Thoracic Surgery, Institut universitaire de cardiologie et de cardiologie de Québec, Quebec City, QC, Canada

P. A. Ugalde, MD

Service of Thoracic Surgery, Université Laval, Quebec City, QC, Canada
In addition, as this secondary breathing circuit is independent of the main ventilation circuit, we can use it at any time during the operation. For example, it can be used when the surgeon wants to either verify an air leak from a bronchial stump or staple line or re-expand other lobes to confirm proper stapler placement (particularly during thoracoscopic cases). This circuit (Bain breathing circuit, Hudson RCI, distributed by Teleflex Medical, Research Triangle Park, NC, USA) can also be used to apply continuous positive airway pressure to the operated lung, although it would be needed infrequently for this purpose. The auxiliary ventilatory circuit can be either a Hyperinflation System (Mercury Medical, Clearwater, FL, USA) or a Bain circuit (Figure) and must be equipped with a manometer to protect the lung from barotraumas.

The advantage of using two independent breathing systems is that they allow us to maintain stable ventilation of the dependent lung during intervention on the operative lung. We believe that an independent breathing circuit is an excellent alternative to clamping the dependent lung during thoracotomy, particularly in this era of increasing video-assisted thoracoscopic surgery where the surgeon frequently asks for "small inflations" of the lung undergoing surgery.

Conflicts of interest None declared.

\section{References}

1. Bruin $G$. Lung re-inflation after one-lung ventilation for thoracic surgery: importance of clamping the dependent lung. Can J Anesth 2014; 61: 1061.

2. Lohser J. One-lung ventilation calls for one-lung recruitment. Anesth Analg 2007; 104: 220. 
3. Jordan S, Mitchell JA, Quinlan GJ, Goldstraw P, Evans TW. The pathogenesis of lung injury following pulmonary resection. Eur Respir J 2000; 15: 790-9.

4. Silva PL, Moraes L, Santos RS, et al. Impact of pressure profile and duration of recruitment maneuvers on morphofunctional and biochemical variables in experimental lung injury. Crit Care Med 2011; 39: 1074-81.

\section{Reply}

Drs. Bussières and Ugalde present an excellent case for using a separate circuit in complex thoracic cases to control inflation and deflation of the operative lung and to test the anastomosis and staple line. Over the last six months we have been using an almost identical approach using a neonatal flow inflating resuscitation circuit (NFIRC) (Figure). These simple circuits are commonly found in obstetrical units as part of the standard resuscitation equipment.

In the majority of our thoracic operations, the lung is relatively healthy and the duration of the surgery is short. Hence, the lung reinflates with low pressures (i.e., $15-20 \mathrm{~cm} \mathrm{H} \mathrm{H}_{2} \mathrm{O}$ ) within $30-45 \mathrm{sec}$ using the original approach that we previously presented (i.e., clamping the lumen to the dependent lung). ${ }^{1}$ The benefits of a separate circuit are not as clear in these cases.

The advantages of a separate circuit are more clearly demonstrated in thoracic decortication cases, where the lung has been extensively and chronically atelectatic and there is other significant lung pathology. It has been suggested that a pressure of $30 \mathrm{~cm} \mathrm{H}_{2} \mathrm{O}$ is acceptable for

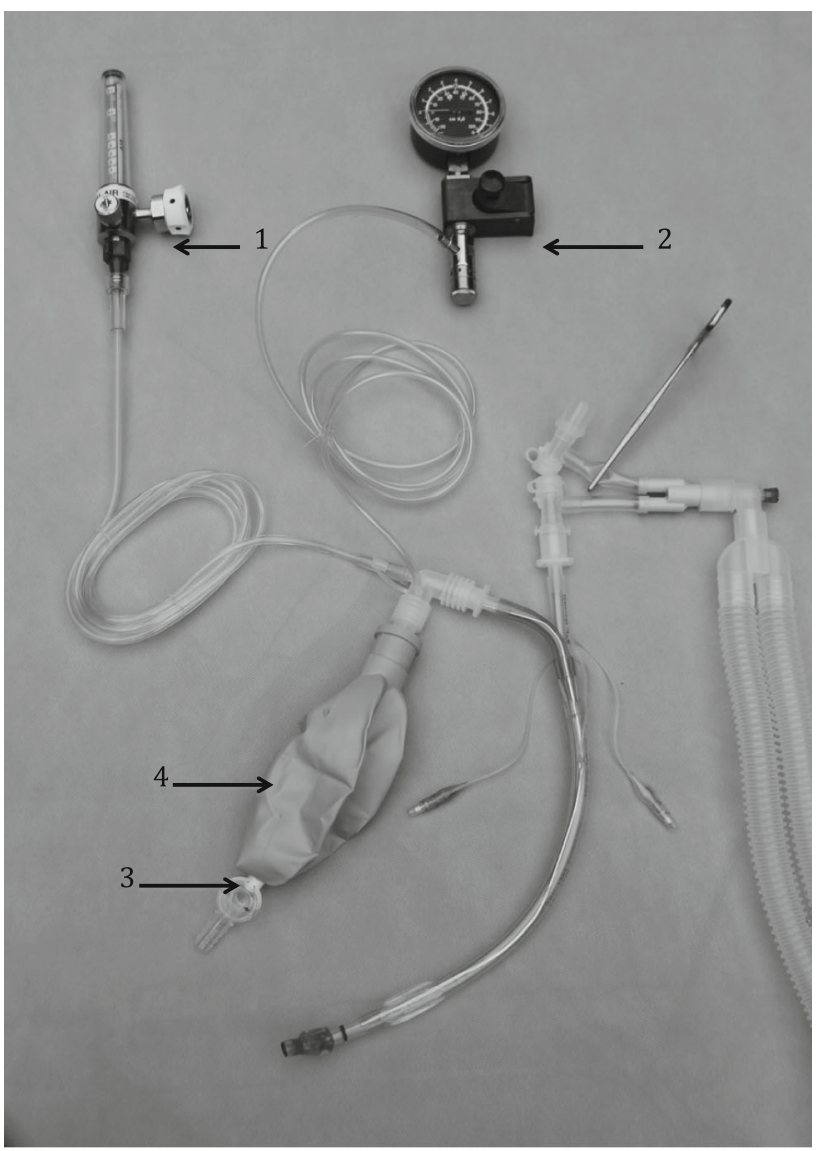

Figure A neonatal flow inflating resuscitation bag is connected to the non-dependent (i.e., operative lung) lumen of a double-lumen endotracheal tube. The anesthesia ventilator circuit tubing is connected to the dependent lumen. 1: medical airflow meter, 2: airway pressure monitor, 3: adjustable pressure-limiting valve, 4: reservoir bag

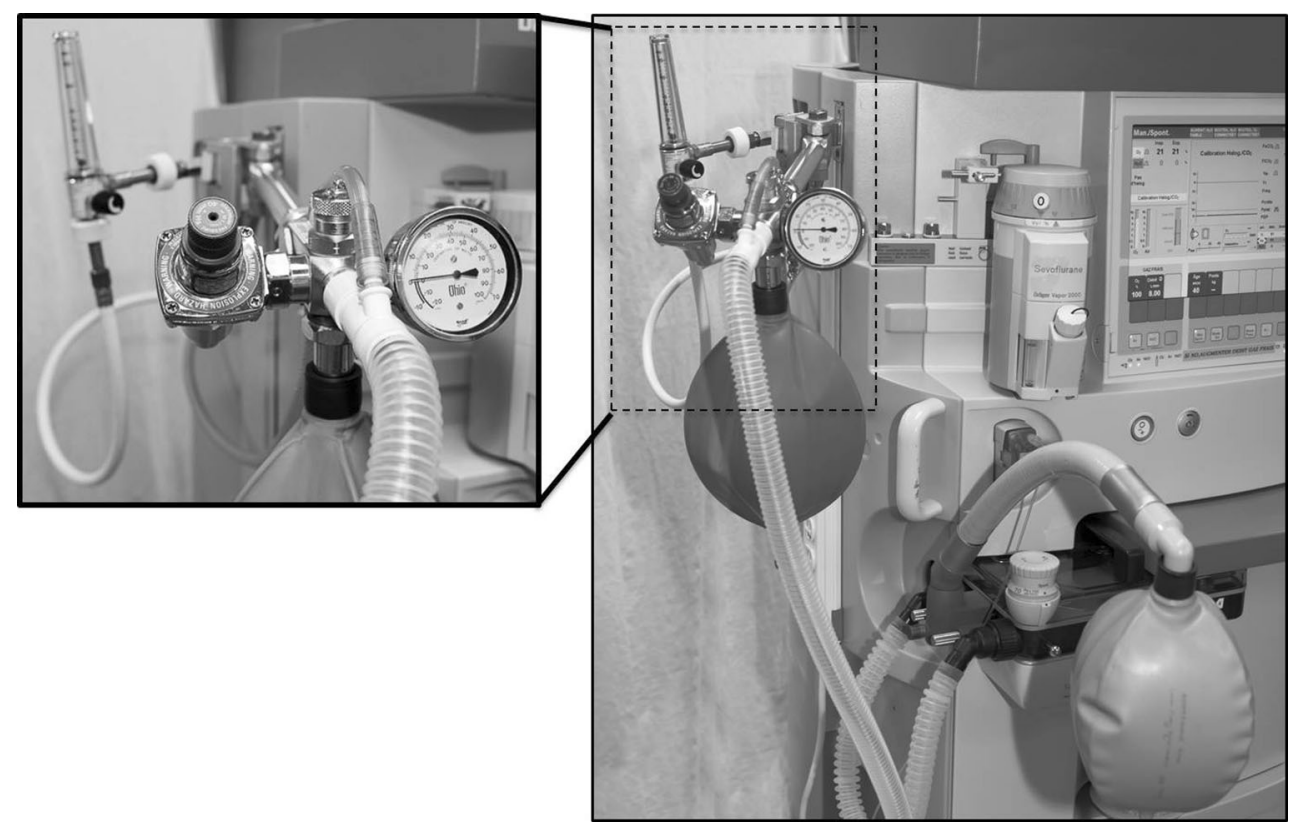

Figure Bain circuit acting as an auxiliary breathing circuit in parallel with the primary circuit to ventilate the non-dependent lung manually 
reinflating the chronically atelectatic lung. ${ }^{2}$ In the case of atelecto-trauma, however, the alveoli that reinflate first do so non-spherically. Thus, a distending pressure of $30 \mathrm{~cm}$ $\mathrm{H}_{2} \mathrm{O}$ can produce alveolar wall stress comparable to that seen with $140 \mathrm{~cm} \mathrm{H}_{2} \mathrm{O} .{ }^{3,4}$ Using the NFIRC, we have been able to extend the duration of reinflation to more than five minutes, usually achieving full inflation with only $15 \mathrm{~cm}$ $\mathrm{H}_{2} \mathrm{O}$ pressure and rarely with more than $20 \mathrm{~cm} \mathrm{H}_{2} \mathrm{O}$. Most anesthesiologists would not want to pause ventilation to the dependent lung for more than two minutes.

With our technique, the NFIRC is attached to the nondependent lumen of the double-lumen tube at the point of starting one-lung ventilation. The reservoir bag of the NFIRC serves as a early warning for loss of lung isolation from outward migration of the DLT. Small amounts of gas passing around the herniated bronchial cuff will exit through the non dependent lumen and cause the reservoir bag to flutter with positive pressure breaths.

When it is time to reinflate the lung, we simply begin by opening the fresh gas flow, set at $10 \mathrm{~L} \cdot \mathrm{min}^{-1}$, to the NFIRC with the adjustable pressure-limiting valve in the open position. The valve is then slowly adjusted toward the closed position to increase the distending pressure. Our preference is not to ventilate the lung manually with the reservoir bag. Instead, we carefully watch the manometer while the lung re-inflates.

Despite using medical airflow for reinflation, we have not seen any reduction in oxygen saturation. Indeed, it usually improves. The main advantage of using this $\mathrm{F}_{1} \mathrm{O}_{2}$ is to prevent absorption atelectasis and repeat atelectotrauma. If a higher $\mathrm{FIO}_{2}$ is desired, the airflow meter can quickly be connected to an adjacent oxygen flowmeter using a $\mathrm{Y}$ connector.

After the lung is re-inflated and surgical manipulation of the lung is complete, we stop ventilating the operative lung. While the surgeon prepares for chest closure, we maintain a continuous positive airway pressure of $5-7 \mathrm{~cm} \mathrm{H}_{2} \mathrm{O}$ to prevent the return of atelectasis.

Thus, we agree with Drs. Bussières and Ugalde in their use of a separate circuit to control inflation of the operative lung in complex thoracic cases. We also offer an alternate method with the widely available NFIRC.

Conflicts of interest None declared.

\section{References}

1. Bruin $G$. Lung re-inflation after one-lung ventilation for thoracic surgery: importance of clamping the dependent lung. Can J Anesth 2014; 61: 1061.

2. Lohser J, Ishikawa S. Clinical management of one-lung ventilation. In: Slinger, editor. Principles and Practice of Anesthesia for Thoracic Surgery. New York: Springer; 2011: 96.

3. Temblay $L N$, Slutsky AS. Ventilator-induced lung injury: from the bench to the bedside. Intensive Care Med 2006; 32: 24-33.

4. Mead J, Takishima T, Leith D. Stress distribution in lungs: a model of pulmonary elasticity. J Appl Physiol 1970; 28: 596-608.

Gerard Bruin MD

Trillium Health Partners, Credit Valley Hospital, Mississauga, ON, Canada 